\title{
ETIENNE CAVALIÉ*
}

\section{LE TYPE NUMISMATIQUE DE SAINT-GILLES}

Résumé - La monnaie comtale de Saint-Gilles, appartenant aux comtes de Toulouse et dont la frappe s'étend sur la première moitié du XII ${ }^{\mathrm{e}}$ siècle, offre un type qui n'a jamais été étudié avec une réelle méthode, si bien que l'interprétation de son image, un quadrupède sommé d'une croix, n'a pas été faite de manière définitive. En intégrant cette monnaie dans des corpus plus vastes, que sont : les représentations de la légende de saint Gilles; les représentations de l'Agneau Pascal des origines au XII ${ }^{\mathrm{e}}$ siècle ; l'ensemble des monnaies seigneuriales du royaume de France, il apparaît que l'animal en question ne peut être qu'un Agneau pascal, ou plutôt un Agnus Dei, figuration de Jésus Christ à la fois comme victime sacrifiée et comme Fils de Dieu ressuscité. Les apparitions de ce type dans divers ateliers européens de manière inattendue montrent que sa propagation n'est pas due à des imitations d'une monnaie à l'autre, mais passe par d'autres supports.

Summary - Stroken during the first half of the 12. century, the figure in the coins of Saint-Gilles, where the money belongs to the counts of Toulouse, has never been studied with real methods, and the interpretation of its quadruped under a cross has not been made for good. But the comparison between this picture and bigger collections (pictures of the legend of St Gilles, pictures of the Holy Lamm, the whole feodal french coins) let no doubt: this animal is an Holy Lamm, which means Jesus Christ as a sacrificed victim and as the ressuscitated Son of God. The unforeseenable apparitions of this picture in many europeans monetary places suggest that its developpment is not owing to imitations between coins, but to some others artifacts.

En publiant en 1860 son deuxième tome sur les Monnaies féodales, Poey d'Avant pense clore le débat portant sur le type monétaire de Saint-Gilles : pour lui, l'animal qui apparaît dans le champ de l'avers est définitivement un Agneau pascal. Or dans la mesure où il s'agit d'un monnayage peu important, aucune étude ne vient le contredire, puisque aucune étude approfondie n'est effectuée, jusqu'à un article de fond de 1956 d'Henri Rolland, qui tranche en faveur d'une théorie rejetée par Poey d'Avant : selon lui, il s'agit d'une biche. Aucun numismate depuis n'a remis sérieusement en cause l'interprétation de Rolland.

\footnotetext{
* Conservateur des bibliothèques. Bibliothèque interuniversitaire scientifique Jussieu, Université Pierre et Marie Curie, 4 place Jussieu, 75005 Paris.
} 


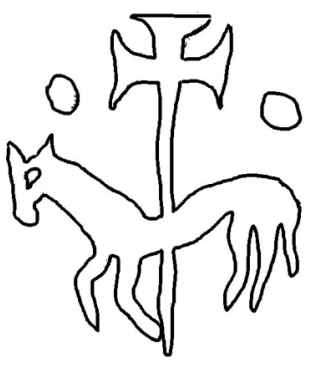

Sur l'histoire du monnayage lui-même, je n'ai pas d'élément nouveau à apporter. Mais le type n'a sans doute pas été suffisamment étudié. Car affirmer que l'animal des monnaies de Saint-Gilles est une biche parce que le quadrupède ressemble à une biche n'est pas un argument suffisant. Il faut encore préciser pourquoi il ressemble à une biche, et surtout : pour qui ? La première question : pourquoi (ou en quoi), amène à se demander comment les biches sont représentées au Moyen Age ; par quels moyens les artisans les identifient. En particulier, il convient de se demander quels sont les moyens qui permettent de distinguer, sans confusion possible, la biche de la légende de saint Gilles des autres biches, ou des autres animaux.

Sans aucun doute, la silhouette évoque plutôt un cheval ou une biche, en tout cas un animal aux membres graciles et au corps fin. Mais il convient encore de démontrer que c'est la silhouette, et plus généralement la forme des objets représentés, des animaux et des personnes, qui est déterminante pour produire du sens dans l'emblématique et la symbolique médiévales.

Il faut reprendre un certain nombre d'éléments épars avec méthode. En soi, le type de Saint-Gilles constitue un motif iconographique extrêmement intéressant. Mais il est également une occasion de développer une méthode pour l'analyse iconographique des types monétaires. Il faut faire appel d'une part à un corpus iconographique rassemblant différents supports, et d'autre part à un certain nombre de textes.

En prélude cependant, il faut insister sur un aspect qui doit être considéré comme primordial pour une étude iconographique: ce qui permet dans l'esprit médiéval d'assimiler une représentation à ce qu'elle représente - par exemple un saint et une 
statue de ce saint - ce n'est pas la ressemblance, mais la présence d'attributs. Ce sont eux qui permettent de distinguer par exemple certains des apôtres parmi le groupe des Douze. La représentation du roi sur les monnaies d'or à partir du XIV ${ }^{\mathrm{e}}$ siècle n'a rien des portraits royaux sur les médailles $\mathrm{du}_{\mathrm{XVI}}^{\mathrm{e}}$ siècle : c'est simplement une figure humaine en pied parée d'un certain nombre d'éléments lui accordant la majesté royale, le dais, la couronne, le sceptre ${ }^{1}$, qui n'a rien d'une tentative de représentation réaliste. Cette question a un rapport très direct avec le type monétaire de Saint-Gilles, comme nous le verrons.

\section{Le monnayage de Saint-Gilles}

En 975, les comtes de Toulouse et les comtes de Rouergue se partagent le comté de Nîmes. Le comte de Toulouse, Guillaume Taillefer (950-1037) reçoit alors Saint-Gilles. Son fils Pons (1037-1060) lui succède comme comte de Toulouse et de Saint-Gilles. Il a lui-même deux fils : l'aîné, Guillaume (1060-1088) reçoit le premier comté ; le second, Raymond Bérenger IV, reçoit Saint-Gilles, puis Toulouse à la mort de son frère en 1088. C'est lui qui crée la moneta egidiensis, signalée pour la première fois dans une charte du 18 février $1096^{2}$.

En 1095, le fils de Raymond Bérenger IV, Bertrand, renonce à Saint-Gilles ${ }^{3}$ à la demande de son père et ne conserve ses droits que sur Toulouse. Son frère AlphonseJourdain naît en 1103 en Terre Sainte, et s'il reçoit probablement le comté de SaintGilles en partage, il n'a aucune force politique lorsque son père meurt en 1105. Bertrand hérite du comté de Toulouse, et occupe celui de Saint-Gilles, à la suite de quoi il est

\footnotetext{
${ }^{1}$ Cf. par exemple le royal d'or de 1328 de Philippe VI (Duplessy 1999, n²47).

2 Arch. dép. Gard, H 785. Le document H 785 est un registre reprenant tous les diplômes relatifs au comté de Saint-Gilles. L'acte est daté du 12 des calendes de mars 1095.

3 Arch. dép. Gard, H 785.
} 
excommunié.

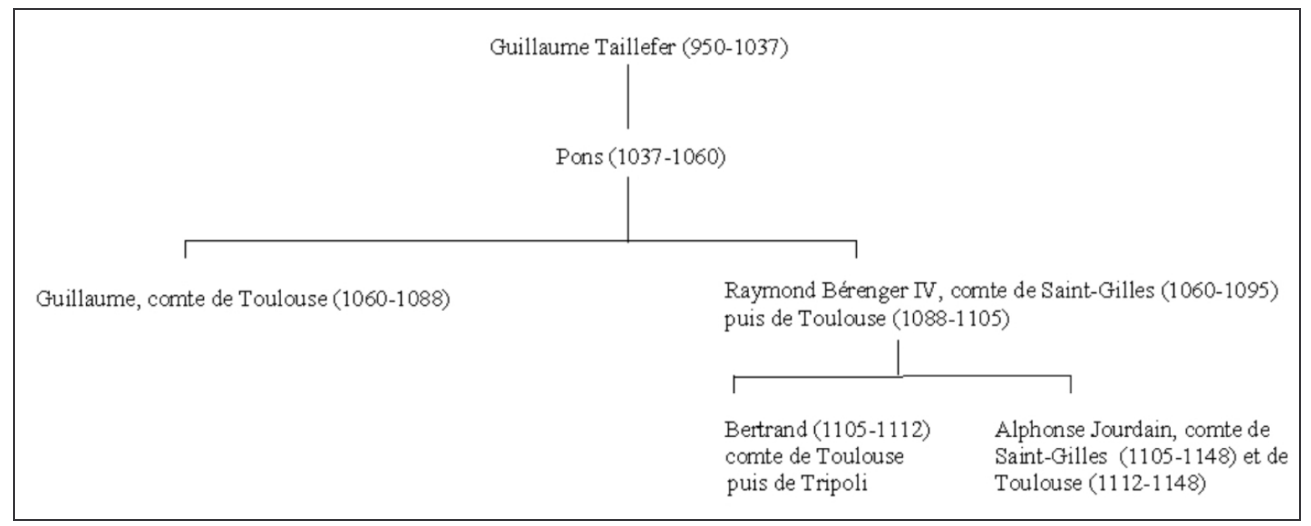

Bertrand part quelques années plus tard avec son fils en Orient, où il prend possession du comté de Tripoli. C'est la raison pour laquelle on retrouve au XIII ${ }^{\mathrm{e}}$ siècle des monnaies émises dans ce territoire qui sont imitées de Saint-Gilles ${ }^{4}$. Lors de cette réapparition à Tripoli, « le quadrupède est bien devenu un Agneau pascal. » ${ }^{5}$

En partant, Bertrand laisse ses terres, dont Saint-Gilles, à son jeune frère AlphonseJourdain, qui devient également comte de Toulouse (1112-1148). C'est apparemment ce comte dont le nom apparaît sur les monnaies : ANFOS COMES.

Des textes signalent la moneta egidiensis en 1105, 1109, 1110, 1138 et 1141 . La dernière mention date de $1144^{6}$, avant la mort d'Alphonse-Jourdain. Mais dès les années 1130-1150, le melgorien réapparaît progressivement comme l'unique monnaie en circulation dans le Languedoc ${ }^{7}$. A partir de 1150, il est en concurrence avec le raymondin frappé par le comte de Toulouse dans le marquisat de Provence. Quoi qu'il en soit, le monnayage de Saint-Gilles est relativement bref (une cinquantaine d'années), et n'a qu'une circulation locale. Le nombre d'exemplaires connus est assez faible. Le

\footnotetext{
4 Alex G. MAlloy, Irene Fraley PREston, A. J. SEltman, Coins of the Crusader States (1098-1291), New York, Attic Books Ltd, 1994, n² 290, p. 175-176.

5 G. SCHLUMBERGER, Numismatique de l'Orient latin, p. 103, pl. IV, 8.

${ }^{6}$ E. DUPRAT, Cartulaire du chapitre de Notre-Dame-des-Doms, Avignon, Musée Calvet, 1932, ${ }^{\circ}$ VII, VIII, XXIX ; E. BALUZE, Histoire généalogique de la maison d'Auvergne, Paris, Dezallier, 1708, t. II, p. 489.

7 M. BOMPAIRE, La circulation monétaire en Languedoc ( $X^{e}-X I I I^{e}$ siècle), thèse de doct., histoire, Paris IV-Sorbonne, 2002, t. III, « Saint-Gilles ».
} 
Cabinet des Médailles de la Bibliothèque nationale de France n'en possède que huit exemplaires : six deniers et deux oboles et pougeoises.

Toutes les monnaies sont de type et de légende identiques, au nom du comte Alphonse ou du comte Raymond (Raymond IV, père d'Alphonse-Jourdain, ou Raymond V, son fils, qui règne de 1148 à 1194). Les seules variations sont : tous les animaux sont tournés vers la gauche, sauf sur un denier où il est à droite ; sur toutes les monnaies la croix est accostée de part et d'autre d'un globule, sauf sur ce même denier et sur une obole. Le dessin et le style sont très similaires d'une pièce à l'autre.

Denier du XII ${ }^{\mathrm{e}}$ siècle :

ONOR SCI EGIDI Quadrupède à g. surmonté d'une croix haute entre deux globules. R. : + ANFOS CONES Croix pattée dans un grènetis.

POEY D’AVANT n 3714, pl. 81, nº 13.

Denier du XI ${ }^{\mathrm{e}}$ ou du XII ${ }^{\mathrm{e}}$ siècle :

ONOR SCI EGIDI Quadrupède à g. surmonté d'une croix haute entre deux globules. R. : + O RAMVNDVS Croix pattée dans un grènetis

PoEy D’Avant n 3718, pl. 81, n 16.

Le mot ONOR est rare dans la numismatique française. Il ne se retrouve que sur les monnaies du Béarn ${ }^{8}$. Son sens a déjà été maintes fois expliqué : il désigne un « domaine ». « Ce mot [honor] est aussi dans le vieux for de Béarn (art. 40 et 90) et dans le Cartulaire de Saint-Pré (de Bigorre, p. 401). [...] Par honor, il faut entendre les droits honorifiques, la seigneurie, le domaine, le territoire, la terre patrimoniale. En Roussillon, les biens immeubles se disaient alaudium, plus souvent alodis ou honor. [...] Ce terme de onor, dont l'origine est espagnole, fut peut-être placé sur la monnaie de Béarn en souvenir de l'acte par lequel Sanche le Grand avait accordé à Centulle le Jeune, vers 1012, la souveraineté du Béarn. » ${ }^{9}$ Une charte de 1095 utilise le mot ONOR

\footnotetext{
8 POEY D’AVANT n ${ }^{\circ} 3233-3243$; RN 1893, p. 475.

9 Gustave SCHLUMBerger, Adrien BlAnCHET, Numismatique du Béarn, Paris, Ernest Leroux, 1893, p. 56.
} 
dans ce sens ${ }^{10}:$ y sont mentionnés les droits de l'honneur de la ville situés à l'intérieur et à l'extérieur de la ville. Ce n'est donc pas un domaine dans sa dimension géographique, mais politique : l'ensemble des droits relevant d'un seigneur ou d'une communauté ${ }^{11}$. L'expression Honor Sancti Aegidii désigne l'ensemble des droits du comte de Saint-Gilles, et la monnaie en fait partie, selon ce que l'affirme la légende des pièces. Le nom propre désigne la seigneurie, non le saint lui-même, ni même l'abbaye homonyme.

Quant à la titulature du comte au revers de la monnaie, bien qu'elle ne pose aucun problème de compréhension, il sera utile de revenir sur sa présence par la suite.

Poey d'Avant fait déjà état d'un débat sur l'interprétation du type du droit. Bien qu'il désigne le type comme étant sans aucun doute possible un «agneau pascal», il mentionne l'hypothèse, qu'il juge non scientifique, d'y voir un cheval, qui serait le signe du comte de Toulouse partant en croisade à la fin du $\mathrm{XI}^{\mathrm{e}}$ siècle. Le cheval n'est désormais plus une hypothèse retenue, les numismates de la seconde moitié du $\mathrm{Xx}^{\mathrm{e}}$ siècle adoptant l'interprétation qu'en fait Henri Rolland en 1956.

Dans un compte-rendu du livre sur la numismatique de l'Orient Latin, Coinage in the Latin East ${ }^{12}$, Michel Dhénin critique le choix du C. J. Sabine qui décrit les monnaies du comté de Tripoli comme ayant pour type «horse and cross $»^{13}$. En effet, il paraît clair depuis le milieu du Xx ${ }^{\text {ème }}$ siècle que «Henri Rolland a démontré que sur les deniers de Saint-Gilles il s'agissait d'une biche. »

Revenons justement sur cette démonstration. Dans son article, Henri Rolland

${ }^{10}$ Dom de VIC, Dom VAISSETE, Histoire générale de Languedoc, t. V, col. 1709.

${ }^{11}$ Du Cange mentionne une occurrence de Honor dans le sens territorium, districtus dans un document de 1357, les Litterae Caroli Johannis Regis primogeniti. Du Cange référence par ailleurs un sens employé en Espagne : feuda, terra, qui se retrouve notamment dans des textes de loi d'Alphonse IX, roi de Castille (1158-1214).

${ }^{12}$ Coinage in the Latin East, The Fourth Oxford Symposium on Coinage and Monetary History, P. W. Edbury et D. M. METCALF éd., Oxford, B.A.R., 1980, IV-148 p. (B.A.R. International Series, 77). 
explique que la forme de la croix n'est pas celle qui accompagne généralement l'Agnus $D e i$, et en conclut que c'est probablement la biche de la légende. Les mots exacts de l'auteur sont les suivants : «Sans insister sur la forme de la croix qui n'est pas celle dont on accompagne généralement l'agneau pascal, il nous apparaît plus vraisemblable de reconnaître dans l'animal passant la biche légendaire qui, dans une forêt déserte, aurait nourri l'ermite Egidius. ${ }^{14}$ La démonstration s'arrête là, et elle ne peut pas être décisive. Cela ne signifie pas que sa conclusion soit fausse, mais qu'il faut relever avant tout les points qui pourraient poser problème. Le premier champ d'investigation concerne l'iconographie de saint Gilles et de sa légende.

\section{L'iconographie de saint Gilles}

Deux questions majeures doivent se poser face à l'hypothèse d'Henri Rolland :

1. la Vie de saint Gilles est-elle déjà composée à la fin $\mathrm{du} \mathrm{XI}^{\mathrm{e}}$ siècle, pour que l'on puisse ainsi s'en inspirer?

2. Quelles sont les représentations qui en existent, et dans quelle mesure s'apparententelles à celle que l'on voit sur les monnaies de Saint-Gilles ?

Comme beaucoup de textes hagiographiques, la Vie de saint Gilles se nourrit à plusieurs sources. Celle qu'a résumée au XIII siècle Jacques de Voragine dans la Légende dorée est tirée du texte français de Guillaume de Berneville, composé en Angleterre vers $1170^{15}$. C'est aussi le récit français le plus ancien de cette Vie. Le seul manuscrit complet qui nous en est parvenu, datant de la première moitié du XIII ${ }^{\mathrm{e}}$ siècle, est actuellement conservé à la Bibliothèque Laurentienne de Florence ${ }^{16}$. C'est un

\footnotetext{
${ }^{13} R N$ 1981, p. 161-162.

${ }^{14}$ Henri Rolland, « La monnaie de Saint-Gilles », Provence historique, t. 5, 1955, p. 1-2.

15 Florence LAUrent, La Vie de saint Gilles de Guillaume de Berneville, Paris, Champion, 2003, introd., p. I.

${ }^{16}$ Florence, Bibliothèque Laurentienne, $\mathrm{N}^{\circ} 99 \mathrm{du}$ fonds des couvents supprimés.
} 
manuscrit composé en Angleterre. Il existe en tout six versions en langue vernaculaire de la Vie :

- Le récit de Guillaume de Berneville

- Deux versions françaises datant du XIV ${ }^{\mathrm{e}}$ siècle.

- Deux versions anglaises, de la même époque.

- Un texte italien de la première moitié du XIV siècle.

On connaît également trois autres textes qui ne retracent que l'épisode de la biche et s'intéressent plus particulièrement à la blessure du saint :

- La Chronique de Mousket (milieu du XIII siècle).

- Une traduction française en prose du texte latin, attribuée à Jean Beleth (seconde moitié du XII ${ }^{\mathrm{e}}$ siècle).

- Une traduction anglaise (fin du XIII ${ }^{\mathrm{e}}$ siècle).

Comme on le voit, le texte de Guillaume de Berneville est le plus ancien de tous, et, daté de 1170 environ, il est postérieur de plus de cinquante ans à l'apparition des monnaies de Saint-Gilles. Toutefois la Vie de saint Gilles a elle-même une source : la Vita sancti Aegidii, publiée par les Bollandistes ${ }^{17}$, et contenue dans un grand nombre de manuscrits composés entre le $\mathrm{XI}^{\mathrm{e}}$ et le $\mathrm{XIV}^{\mathrm{e}}$ siècle. Une des versions connues aurait été rédigée au $\mathrm{X}^{\mathrm{e}}$ siècle $^{18}$.

Le culte de saint Gilles, dont la fête se situe au $1^{\text {er }}$ septembre, est attesté dès le IX ${ }^{\mathrm{e}}$ siècle. Il est mentionné par Usuard dans son Martyrologe, daté des alentours de $875^{19}$. Au sein de l'abbaye où se trouve son corps, ce culte est au moins antérieur à 924 : c'est l'époque où l'abbaye le choisit comme saint patron. Il faut préciser que l'existence du monastère lui-même est attestée dès 878 , dans deux bulles pontificales faisant référence

17 Acta Sanctorum, Sept., t. I, p 299-303.

18 On trouve des extraits de cette rédaction dans l'Office composé par Fulbert de Chartres, mort en 1023, en l'honneur de saint Gilles. Cf. Patrologie Latine, t. CLXI, p. 343.

${ }^{19}$ Le Martyrologue d'Usuard, J. DuBoIs O.S.B. éd., Bruxelles, 1965. 
à un acte de 685 .

Le texte latin contenant l'épisode de la biche et du saint est donc effectivement antérieur à l'apparition des monnaies, et fort répandu lorsque Alphonse-Jourdain devient comte de Saint-Gilles. Voilà donc la première question résolue.

Le contenu du récit n'est pas à négliger, car il nous apprend que d'autres épisodes que celui de la biche entretiennent le culte de saint Gilles. Par ailleurs la Vita permet de concevoir quelles représentations peuvent émaner de la scène de la chasse.

Gilles, Athénien d'origine, part en Gaule et vit durant trois ans dans une forêt de Septimanie, se nourrissant de racines et d'herbes crues, ainsi que du lait d'une biche envoyée par Dieu. Un jour le roi Flavius vient chasser dans la forêt. La biche échappe par deux fois aux chiens, qui refusent de s'approcher des fourrés où l'animal s'est réfugié. Pour faire sortir la bête, un chasseur tire une flèche qui atteint saint Gilles et le blesse gravement. Les chasseurs se fraient un chemin dans les broussailles et découvrent un vieil homme aux cheveux blancs, avec la biche allongée à ses pieds. Le roi s'approche alors de l'ermite, l'interroge, lui demande pardon pour la blessure infligée et lui propose des remèdes. Mais le saint refuse leur aide et demande même à Dieu, après leur départ, de ne jamais guérir sa blessure. Par la suite, le saint conseille au roi de faire construire un monastère, et en devient l'abbé à la demande du souverain.

Les martyrologes et les chroniques du $\mathrm{XII}^{\mathrm{e}}$ au $\mathrm{XIV}^{\mathrm{e}}$ siècle situent la vie du saint en 700-740. Selon Florence Laurent, « les seuls épisodes dont l'authenticité historique n'est [pas] contestée [...] concernent l'existence du roi Flavius, titre pris par les rois wisigoths d'Espagne, et le voyage à Rome du saint. ${ }^{20}$ La correspondance des deux permet d'identifier le pape comme Benoît II (684-685), et le roi comme Wamba, qui assiègea Nîmes en 673. 
Il est indispensable de rapporter ici un autre épisode de cette Vie, qui rendit le saint extrêmement sympathique dans la ferveur populaire. Il concerne un Carolingien, tantôt Charles Martel, tantôt Charlemagne : l'un ou l'autre de ces deux hommes ayant commis un péché tellement horrible qu'il n'ose s'en confesser, un ange apporte à saint Gilles une cédule sur lequel est écrit ce péché, tandis que l'abbé est en train de dire une messe. A mesure que Gilles prononce ses prières, les mots s'effacent du document.

A cause de cela, saint Gilles ne concerne pas seulement les chasseurs, mais aussi les pécheurs, car il permet d'obtenir le pardon de Dieu sans avoir à passer par la confession (la rémission demandée est accordée à condition de ne pas commettre une seconde fois la même faute). C'est surtout pour cette dernière raison qu'il est l'objet d'un culte important au Moyen Age.

On trouve ainsi deux représentations essentielles de saint Gilles :

- Gilles protège une biche poursuivie par des chasseurs.

- Gilles célèbre une messe en accordant l'absolution au roi.

Cet aspect est important à prendre en considération, car l'esprit moderne a trop tendance à retenir de cette Vita l'histoire de la biche pourchassée, tandis que le culte qui est rendu au saint est plutôt motivé par le second épisode. Dans les représentations du saint en pied, comme patron d'une chapelle dans une église par exemple, la biche n'est nullement un attribut nécessaire : saint Gilles et sa biche ne sont pas aussi inséparables que saint Roch l'est de son chien.

Il n'est pas forcément aisé de trouver des représentations scéniques de saint Gilles et de sa vie. L'église abbatiale de Saint-Gilles n'en comporte pas de représentation ${ }^{21}$. La châsse de saint Gilles des $\mathrm{XI}^{\mathrm{e}}$-XII ${ }^{\mathrm{e}}$ siècles, aujourd'hui perdue (mais décrite dans le

\footnotetext{
${ }^{20}$ F. LAURENT, op. cit. n. 15, introd., p. XXIV.

21 Rien de tel du moins n'apparaît dans MAGUELONE, Saint-Gilles et son abbatiale, Paris, Barre et Dayer, 1990, 102 p.
} 
fameux Guide du pèlerin de Saint-Jacques-de-Compostelle), n'en contenait pas non plus $^{22}$.

Une petite liste est fournie par l'ouvrage de Louis Réau ${ }^{23}$. D'autres exemples ont pu être trouvés en interrogeant les bases iconographiques du Ministère de la Culture accessibles par Internet : Bases Mérimée, Palissy ${ }^{24}$; ainsi que les bases d'enluminures médiévales Liber Floridus ${ }^{25}$ et Enluminures ${ }^{26}$.

Plusieurs éléments s'en dégagent. Lorsque l'on trouve le saint seul, plusieurs attributs l'accompagnent, soit simultanément, soit alternativement: essentiellement une biche, un lis $^{27}$, une crosse. Il convient d'insister sur ce dernier élément, car saint Gilles est beaucoup plus souvent représenté en abbé qu'en ermite, et ce notamment lorsqu'il est au milieu d'autres saints ${ }^{28}$.

La représentation de la biche elle-même est assez réaliste. Cela s'explique surtout par le fait qu'elle apparaît presque toujours dans un décor réaliste : ce n'est pas l'animal en tant que tel qui est représenté, mais la scène de la chasse de la Vita, ou un ermite dans les bois avec cet animal : elle n'est qu'un des éléments de l'ensemble, elle n'est pas un emblème du saint à elle seule. Elle n'est donc pas stylisée, mais intégrée dans le décor. Elle a une petite queue dressée en l'air, ou pas de queue du tout.

Ajoutons que la biche peut être représentée en tant qu'attribut de saint Gilles. Mais si elle doit apparaître seule, comme ce serait le cas sur les monnaies de Saint-Gilles, elle doit alors être elle-même dotée d'attributs : ce seraient assez naturellement une flèche,

\footnotetext{
22 Dr PETOURAUD, L'iconographie de la châsse de saint Gilles, Lyon, 1949.

23 Louis REAU, Iconographie de l'art chrétien, t. III : Iconographie des saints, part. II : G-O, Paris, PUF, 1958, 1019 p.

24 Ministère de la Culture, Bases Mémoires, [En ligne] http://www.culture.gouv.fr/public/mistral/memoire_fr (consulté le 5 juin 2005).

25 Centre informatique national de l'Enseignement supérieur, Liber Floridus, [En ligne], http://liberfloridus.cines.fr/ (consulté le 3 juin 2005).

26 Direction du Livre et de la Lecture, CNRS, Enluminures, [En ligne], http://www.enluminures.culture.fr (consulté le 3 juin 2005).

${ }^{27}$ Les peintres italiens accompagnent saint Gilles, Gilio (prononciation : Giglio), d'un lis, à cause de l'homophonie avec le terme italien désignant le lis.
} 
ou un chien la poursuivant. Et si l'on considère le programme iconographique de saint Gilles dans les églises de Largny-sur-Automne, et Saint-Nicolas de Civray (peintures murales $)^{29}$, il n’y a nullement de présence de croix dans la scène de la chasse.

Deux observations se dégagent de ces comparaisons avec l'iconographie de saint Gilles :

1. D'une part, il apparaît que, pour représenter saint Gilles, nul n'est besoin de la biche : le saint lui-même a ses propres attributs.

2. D'autre part, aucune croix n'apparaît sur les scènes de la chasse de la biche : elle n'est nullement un attribut, ni de saint Gilles, ni de sa biche. Et s'il en était une, on s'expliquerait difficilement sa disposition, sur les monnaies qui nous occupent, en travers du corps de la biche (derrière elle, en fait) et non pas à côté.

La présence de cette croix crée alors une confusion avec un autre animal, représenté plusieurs milliers de fois dans l'iconographie médiévale : l'Agnus Dei. C'est la figure qu'il nous faut à présent étudier.

\section{L'iconographie de l'Agneau de Dieu}

Voici d'abord les éléments qui rendent difficile l'assimilation de l'animal de saint Gilles à l'animal christique :

- Le quadrupède représenté ne ressemble en rien à un agneau. Sa silhouette est très fine, non laineuse. Son cou est trop long.

- Il n'est pas paré d'un nimbe.

- Il ne retourne pas la tête vers l'intérieur.

- Il est entouré de la légende : HONOR SCI EGIDII, et d'une part cela invite à chercher dans le type une allusion au saint mentionné en légende; d'autre part sur la très grande majorité des représentations de l'Agnus Dei, celui-ci est entouré des mots : «ECCE AGNVS DEI QVI

\footnotetext{
${ }^{28}$ Par exemple : Paris, Bibl. Mazarine, ms. 0491, fol. 260v.

${ }^{29}$ Les images sont accessibles sur la base Mérimée du Ministère de la Culture. La faible qualité des images téléchargeables en a rendu impossible la reproduction ici.
} 
TOLLIT PECCATA MVNDI » ou une expression voisine, qui désigne clairement l'agneau comme représentation du Fils de Dieu.

- Il n'a aucune raison d'apparaître soudainement à Saint-Gilles, sans qu'aucune influence extérieure dans un monnayage voisin puisse expliquer cette création ex nihilo. En revanche, une allusion à la légende du saint patron s'expliquerait fort bien.

La représentation du Christ en agneau apparaît très tôt dans l'histoire $d u$ christianisme. Elle trouve ses sources aussi bien dans l'Ancien que dans le Nouveau Testament.

Des textes bibliques, ne citons ici que les principales mentions. Dans le livre d'Isaïe, le Messie attendu est comparé à «la brebis conduite à la boucherie » et à «l'agneau muet sous la main de celui qui le tond. ${ }^{30}$ Dans l'Evangile selon saint Jean, Jean le Baptiste, en plongeant Jésus dans le Jourdain, déclare : «Voici l'agneau de Dieu, voici celui qui ôte le pêché du monde. ${ }^{31}$ Il n'explique pas cette formule, qui semble claire pour ses auditeurs, probablement parce qu'elle fait référence à une même pratique des textes bibliques ou para-bibliques et de la liturgie: c'est une allusion au sacrifice quotidien de deux agneaux au Temple (matin et soir), exigés dans le livre de l'Exode ${ }^{32}$. Dans l'Apocalypse enfin ${ }^{33}$, Jean voit l'agneau de Dieu au ciel dans la gloire devant le trône, à l'égal de Dieu ; debout, comme immolé, il n'est pas égorgé, il est vivant, mais porte les nobles cicatrices des blessures qui lui ont donné la mort. Il a la puissance de rompre les sceaux du livre mystérieux.

La représentation de l'agneau évoque donc aussi bien la mission du Christ sur terre, sa mise à mort et sa résurrection que, à travers le texte de l'Apocalypse, son retour à la fin des temps pour le Jugement dernier. Sa signification est extrêmement puissante,

\footnotetext{
30 Is, 53,7 .

$31 \mathrm{Jn}, 1,29$.

32 Ex, 29, 38-46.
} 
autant qu'elle est multiple.

Commençons par faire une distinction entre les deux expressions, «Agneau pascal » et «Agneau de Dieu». L'Agneau pascal est l'agneau consommé lors de la fête de la Pâque. L’Agneau de Dieu, ou Agnus Dei, est une représentation imagée du Christ sous les traits d'un agneau. Toutefois, je ne ferai ici pas de distinction parce que l'usage est clairement établi d'utiliser les deux expressions pour désigner l'Agnus Dei.

La mort du Christ sur la croix est très tôt apparentée au sacrifice de l'agneau. Le thème est très fréquent, et désigne à la fois la Crucifixion et la Résurrection, à tel point que la croix dont on se met progressivement à munir l'Agneau est peu à peu appelée «Croix de Résurrection » ${ }^{34}$. C'est la croix que, sur certaines représentations, le Christ tient dressée en sortant de son tombeau. On en trouve un exemple sur le tombeau du pape Nicolas III (1277-1280), conservé sous la basilique Saint-Pierre de Rome.

L'animal fournit donc une symbolique extrêmement riche, et ceux qui l'utilisent dans leurs mosaïques ou leurs fresques ne cherchent pas nécessairement à préciser le sens qu'ils emploient. «Les anciens paraissent avoir confondu l'agneau, la brebis, le mouton, qu'ils ont pris indifféremment pour symboliser le Christ ou les fidèles », y compris dans les différentes traductions de la Bible ${ }^{35}$. Le symbolisme primitif utilise d'abord l'agneau comme une représentation de la victime expiatoire du Golgotha ${ }^{36}$. Il reçoit ensuite les attributs du Bon Pasteur. On en vient donc à représenter un agneau qui est lui-même un berger.

\footnotetext{
33 Ap, 5, 6.

34 Il est possible de retracer l'apparition progressive de cette croix haute comme attribut de l'Agneau, mais non l'appellation de «Croix de Résurrection», qui n'est pas analysée dans les Dictionnaire de théologie catholique, Dictionnaire d'archéologie chrétienne et de liturgie, et Dictionnaire de la spiritualité. Elle est aussi appelée «croix-étendard», ou «croix vexillifère », ou «étendard crucigère » lorsqu'à la croix est associé un fanion. L'appellation singulièrement paradoxale de «Croix de Résurrection » mériterait pourtant une étude approfondie. Cf. Jacky LEGGE, «Symboles funéraires, abréviations, imageries de nos cimetières », Vivat, s.d., [En ligne] http://www.vivat.be/0000.asp?articleID=657 (consulté le 23/09/2005).

${ }^{35}$ Dictionnaire de théologie catholique, t. I, col. 878.

${ }^{36}$ Cf. Dictionnaire de théologie catholique, t. I, fig. 195-196.
} 
F. Nikolash ${ }^{37}$, qui référence les citations des Pères sur l'Agneau comme symbole christique $^{38}$, distingue trois symboliques essentielles de l'Agneau pascal :

- Symbole de la Passion du Christ.

- Symbole de l'humanité du Christ.

- Symbole de l'Eucharistie.

Curieusement, il ne mentionne pas l'Agneau comme symbole du Christ ressuscité. Les Pères glosent la métaphore de l'agneau de Dieu à l'envi, et vont même chercher dans les textes bibliques des désignations non explicites. La liturgie reprend cette métaphore, notamment lors de la célébration du Jeudi Saint.

La représentation du Christ en Agneau, apparue au $\mathrm{IV}^{\mathrm{e}}$ siècle, finit par paraître gênante, voire avilissante, pour l'Eglise orientale. En 692, le concile Quinisexte des églises orientales décrète qu'il faut remplacer l'Agneau par une représentation anthropomorphe. A partir de cette date, dans l'Eglise grecque, ce motif n'est plus toléré. Cette décision ne l'empêche pas de continuer à se développer en Occident.

Il existe une iconographie assez abondante, qui évolue au fil des premiers siècles de la chrétienté. Au début, l'agneau est représenté portant les attributs du Bon Pasteur. C'est un ornement symbolique sur beaucoup de monuments chrétiens en Orient et en Occident. Afin de rendre l'allégorie plus sensible, on donne à l'agneau des attributs de plus en plus significatifs et clairs du Sauveur ${ }^{39}$ :

- Premiers siècles : avec les insignes du Bon Pasteur : vase de lait, bâton pastoral $^{40}$.

- IV $^{\mathrm{e}}$ siècle : Agneau sur un monticule d'où coulent quatre fleuves. Sur une autre représentation, un agneau agit à la place du Christ, ressuscite Lazare,

${ }^{37}$ F. NiKOLASH, Das Lamm als Christussymbol, Vienne, 1963.

${ }^{38}$ Il ne s'intéresse malheureusement pas à l'iconographie.

${ }^{39}$ Les exemples suivants sont donnés par le Dictionnaire de théologie catholique, Paris, 1930, t. I, part. 1, col. 579. 
multiplie les pains.

- $\mathrm{V}^{\mathrm{e}}$ siècle : Agneau avec une tête nimbée ${ }^{41}$. Plus tard, il reçoit un nimbe crucigère $^{42}$ ou monogrammatique ${ }^{43}$.

- $\mathrm{VI}^{\mathrm{e}}$ siècle : Agneau souffrant la Passion, ou avec des attributs évoquant la Passion.

- A la même époque, on voit apparaître l'agneau glorieux : avec un étendard ou une croix vexillifère; avec une ceinture d'or; armé d'une croix, repoussant un serpent.

- VIII ${ }^{\mathrm{e}}-\mathrm{IX}^{\mathrm{e}}$ siècles : la vision de l'Apocalypse est reproduite dans des mosaïques.

Par la suite, le Moyen Age n'apporte que des modifications de détail. L'utilisation des attributs se fait de plus en plus systématique et appuyée.

Sur les colonnes antérieures du ciborium de Saint-Marc à Venise, on voit une croix grecque, un disque chargé d'un agneau passant à gauche brochant sur le cœur; la Vierge et saint Jean au pied de la croix ; les larrons crucifiés de part et d'autre. C'est là une des représentations les plus anciennes de l'Agneau dans un disque ${ }^{44}$.

Un des supports où on le rencontre très fréquemment, pour une raison sur laquelle nous reviendrons, est le sceau ${ }^{45}$.

Certains animaux sont utilisés par tous les détenteurs de sceaux ${ }^{46}$, d'autres essentiellement ou exclusivement par les seigneurs laïques ${ }^{47}$. L'Agneau est un animal caractéristique des sceaux ecclésiastiques.

${ }^{40}$ Roller, Les catacombes de Rome, Paris, 1879, t. I, p. 249, fig. 9.

${ }^{41}$ Mosaïques de Saint-Jean-de-Latran et Sainte-Pudentienne.

42 Sarcophages d'Aix et Saint-Maximin. Cf. E. Le Blant, Les sarcophages de la Gaule, Paris, 1886, p. 145 , n. 207, pl. LII, n. 2 ; p. 156, n. 214, pl. LVI, n. 1.

${ }^{43}$ R. GARUCCI, Storia dell'arte cristiana, Prato, 1872, t. I, p. 235, n. 1 ; pl. LVIII, n. 1.

44 A. VENTURI, Storia dell'arte italiana, Milan, 1901, t. I, p. 450 et fig. 261.

45 Yves Metman, Michel Pastoureau, «La faune dans les sceaux du Moyen Age », dans Le Bestiaire des monnaies, des sceaux et des médailles, Paris, Hôtel de la Monnaie, 1974, p. 179-239.

${ }^{46}$ Par exemple : le lion, le cerf. 
Lors de la représentation d'animaux dans les armoiries et/ou les sceaux, on trouve deux cas :

- l'animal est représenté seul.

- L'animal fait partie d'une scène d'ensemble.

«Dans le premier cas, les graveurs de sceaux appliquent avec une rigueur progressive les lois de la schématisation héraldique. Dans le second cas, l'artiste, participant au goût de son temps pour l'observation de plus en plus exacte de la nature, introduit des détails de plus en plus précis sur l'anatomie ${ }^{48}$. L'Agnus Dei appartient au premier groupe.

L'exposition de 1974 à l'Hôtel de la Monnaie consacrée aux animaux sur les monnaies, sceaux et médailles, compte trois sceaux montrant un Agnus Dei :

- Ville de Béziers, 1226 (74 mm $)^{49}$.

- Ville de Toulouse, $1242(90 \mathrm{~mm})^{50}$.

- Ville de Narbonne, $1243(70 \mathrm{~mm})^{51}$.

L'Agneau pascal est très fréquent au revers des sceaux ecclésiastiques, c'est-à-dire sur les contre-sceaux : les sceaux ecclésiastiques sont fréquemment en fuseau, mais leurs contre-sceaux sont généralement ronds. On le trouve également au revers des sceaux de villes du Languedoc : les trois exemples ci-dessus le confirment. Il subsiste encore dans les armes de Perpignan, Carcassonne, Toulouse, Auch. L'Agneau pascal est choisi par le chapitre des nobles de Toulouse. Il est alors repris sur les poids; dans cette ville, il tient une croix cléchée, vidée, pommelée (la « croix occitane »).

A Narbonne, l'agneau est nimbé, et il tient une croix pattée. On connaît quatre sceaux de Narbonne conservés : on peut y constater que le corps de l'animal est de plus en plus réaliste.

A Saint-Gilles même, parmi d'autres institutions religieuses, se trouve dès la première

\footnotetext{
${ }^{47}$ M. PASTOUREAU, op cit. $n .46$, p. 183.

${ }^{48}$ M. PASTOUREAU, op cit. $n .46$, p. 184.

49 Arch. nat., D 5614 bis.

${ }^{50}$ Arch. nat., D 5683 bis.

51 Arch. nat., D 5651 bis.
} 
moitié du XII ${ }^{\mathrm{e}}$ siècle un prieuré de l'Ordre de Saint-Jean de Jérusalem. Cet ordre des Hospitaliers apparaît officiellement, avec reconnaissance de la Papauté, en 1113, et dès cette époque il dispose d'un Grand Sceau. Le plus ancien sceau connu du prieuré de Saint-Gilles $^{52}$ date de 1140-1142. Il est appendu à la Harley Charter ${ }^{53}$. Il est circulaire et représente le prieur agenouillé de profil, mains jointes, avec en légende: + ARNALDVS HOSPITALIS S EGIDII PRIOR. Il n'a pas de contre-sceau. En revanche dans deux sceaux plus tardifs, en 1255 et $1271^{54}$, si le sceau reste le même (avec une légende différente, anonyme: + : SIGILLVM : PRIORIS : SANCTI . EGIDII), un contre-sceau est apparu, montrant un Agnus Dei à gauche tenant une croix, non nimbé ${ }^{55}$, avec pour légende circulaire : S PROPATVS SCI EGIDII HOSPITAL S IOHIS IERLEM ${ }^{56}$.

Le dessin de ce sceau est beaucoup plus «canonique» que celui de la monnaie de Saint-Gilles. Il est également plus tardif. Or ce type n'apparaît que rarement sur les sceaux des Hospitaliers: sur tous les sceaux recensés par E. King, seuls trois autres montrent également un Agneau pascal ${ }^{57}$. Les types sigillaires de l'Ordre les plus fréquents sont saint Jean-Baptiste, le Grand Maître (ou le prieur du prieuré) agenouillé, ou la Dormition de la Vierge. Or un Agneau pascal apparaît précisément à Saint-Gilles. Dans le sud de la France, ce thème iconographique est particulièrement « dans l'air » ${ }^{58}$.

Comme on l'a vu, les attributs de l'Agneau évoluent au fil des siècles, tendant à renforcer l'assimilation de l'animal avec le Christ. Lorsque débute le Bas Moyen Age

\footnotetext{
52 E. J. KING, The Seals of the Order of St.-John of Jerusalem, Londres, Methuen, 1932, p. 46-48, pl. XV.

53 British Museum, 83, C, 41.

54 Aucun sceau n'est malheureusement connu entre 1140 et 1255.

55 On peut aussi noter qu'il a une queue longue.

${ }^{56}$ E. J. KING, op. cit. n. 53, pl. VIII, 5-6.

${ }^{57}$ Celui du prieuré de Corbeil, sur un acte de 1233 (E. J. KING, op. cit. n. 53, pl. IX, 9), et deux sceaux en fuseau appendus à une indulgence de 1410 (E. J. KING, op. cit n. 53., pl. XX, 5-6).

${ }_{58}$ Michel DhEnIn, «L'Agnus Dei, thème monétaire », dans Le Bestiaire des monnaies, des sceaux et
} 
cependant, l'image la plus récurrente est relativement stable, «classique ». L'Agneau pascal est alors identifié de la manière suivante :

- Quadrupède, éventuellement recouvert d'une toison bouclée.

- Sa tête est souvent retournée vers l'intérieur, surtout lorsque l'animal est dessiné avec un certain souci de réalisme; et il a une patte avant repliée sur le pied de la croix.

- Se trouve derrière lui (rarement devant), verticalement, une houlette sommée d'une croix, ou d'un étendard, ou des deux.

- L'ensemble de cette figure se trouve dans un cercle.

Les attributs réellement systématiques sont les suivants : la croix de résurrection (dans toutes ses variantes); le cercle dans lequel s'inscrit l'Agneau pascal. J'ai déjà parlé de la croix de résurrection. Le second attribut, le cercle, n'est curieusement jamais relevé par les historiens de l'art et les iconographes. Le nimbe a différentes formes ; la croix également; l'animal lui-même n'a pas une silhouette très stabilisée (j'y reviendrai), et la plus grande permanence dans la figure est en fin de compte la suivante : l'Agnus Dei est circonscrit dans un cercle.

Avant d'expliquer l'origine de cet «attribut», il convient d'en tirer deux remarques : 1. sur des représentations où le support est large (une fresque, une abside, un manuscrit), le graveur ou le peintre dessine malgré tout un $\operatorname{cercle}^{59} ; 2$. lorsque le support est rond par nécessité ou habitude, on y place presque spontanément un agneau. Cela se voit particulièrement: sur les clés de voûte, les patènes, les sceaux, et nombre d'objets d'orfèvrerie. L'origine de ce trait est la suivante: on retrouve systématiquement cette figure sur certains objets, les Agnus Dei pascals.

«On appelle de ce nom certaines bulles, ou certains médaillons de cire empreints de

des médailles, Paris, Hôtel de la Monnaie, 1974, p. 164.

${ }^{59}$ Cf. fig. 7, un linteau de marbre gravé d'une inscription avec un Agneau, datant des années 1100 et scuplté en Languedoc. Les exemples sont inombrables. 
la figure d'un agneau portant la croix-étendard ${ }^{60}$ distribués le dimanche de Pâques par le pape. L'apparition du phénomène liturgique serait contemporaine de l'apparition du cierge pascal au $\mathrm{IV}^{\mathrm{e}}$ siècle, voire antérieure. Ces médailles évoluent et prennent la forme de médaillons avec un agneau aux alentours du $\mathrm{VI}^{\mathrm{e}}$ siècle. Ces objets sont bénis puis distribués aux fidèles au cours d'un cérémonial spécifique, au lendemain de la Veillée pascale. Sur ce cérémonial, il n'existe cependant aucun texte antérieur au IX $X^{\mathrm{e}}$ siècle. Les plus anciens sont l'Ordo romanus ${ }^{61}$ et le traité d'Amalaire ${ }^{62}$. Le Glossaire archéologique affirme que ces objets « figurent au Moyen Age parmi les objets de piété que les orfèvres exécutèrent avec le plus de recherche, d'élégance et de goût. » ${ }^{63}$ Je n'ai pu retrouver ces objets d'orfèvres. En revanche il est certain que les originaux de cire, très fragiles, sont rarement conservés aujourd'hui ${ }^{64}$, et que ce qu'on ne peut étudier le plus souvent ne sont que leurs reproductions, dessinées ou ouvragées.

A cause de cette pratique liturgique, l'image elle-même de l'Agneau est associée rapidement à la forme qui le contient: le cercle. C'est non pas seulement l'Agneau pascal, mais l'Agneau pascal dans un cercle, qui constitue alors une image se répandant largement dans l'Europe occidentale chrétienne.

\section{Les numismates et le type de Saint-Gilles}

Après avoir reconstitué un large décor, revenons à des préoccupations plus proprement numismatiques.

Le thème de l'Agneau pascal est interdit en Orient en 692, mais cela n'empêche pas son adoption et sa propagation en Occident, y compris sur les monnaies.

\footnotetext{
${ }^{60}$ Dictionnaire d'archéologie chrétienne et de liturgie, t. I, «Agnus Dei ».

61 Patrologie Latine, t. LXXVIII, col. 960-961.

62 AMALARIUS, De ecclesiasticis officiciis, dans la P.L., t. CVI, col. 1033 ; t. CI, col. 1215.

${ }^{63}$ V. GAY, Glossaire archéologique, Paris, 1887, p. 11.

${ }^{64}$ On peut en trouver quelques exemplaires dans les Musées du Vatican, en mauvais état de conservation.
} 
Michel Dhénin ${ }^{65}$ recense vingt-quatre lieux (excluant Saint-Gilles) où est frappé le type de l'Agneau, s'étalant du XI ${ }^{\mathrm{e}}$ au XVIII ${ }^{\mathrm{e}}$ siècle. Parmi ceux-ci, les ateliers frappant à ce type entre la fin du $\mathrm{XI}^{\mathrm{e}}$ et la fin du XIII ${ }^{\mathrm{e}}$ siècle sont :

- La Bohême (v. 1100) ${ }^{66}$.

- Naples et la Sicile (milieu XII ${ }^{\mathrm{e}}$ siècle) ${ }^{67}$.

- Le comté de Namur (XII ${ }^{\mathrm{e}}$ siècle $)^{68}$.

- Tripoli (seconde moitié du XII ${ }^{\mathrm{e}}$ siècle) ${ }^{69}$.

- Bressanone $\mathrm{B}^{70}$ (seconde moitié du XII ${ }^{\mathrm{e}}$ siècle).

- Saint-Gall (XIII siècle $)^{71}$.

- Alsace (milieu du XIII ${ }^{\mathrm{e}}$ siècle) $)^{72}$.

- Hongrie (milieu du XIII ${ }^{\mathrm{e}}$ et début du XIV ${ }^{\mathrm{e}}$ siècle) ${ }^{73}$.

- Silésie (milieu du XIII ${ }^{\mathrm{e}}$ siècle) $)^{74}$.

- Tirlemont, auj. Thienen $\left(\mathrm{XII}^{\mathrm{e}}-\mathrm{milieu} \mathrm{du} \mathrm{XIII}^{\mathrm{e}} \text { siècle }\right)^{75}$.

- Orient latin (seconde moitié du XIII ${ }^{\mathrm{e}}$ siècle) ${ }^{76}$.

- Meissen (seconde moitié du XIII ${ }^{\mathrm{e}}$ siècle) ${ }^{77}$.

- Neisse $^{78}$ (v. 1300).

${ }^{65}$ Michel DHENIN, op. cit. n. 60, p. 163-177.

66 František CACH, Nejstaršì Cešké Mince, Prague, Ceská Numismatická Spolecnost, 1972, t. II, $\mathrm{n}^{\circ}$ 439. Monnaie de Svatopluk (1095-1107); Edmund KOPICKI, Katalog podtsawowych typóv monet $i$ banknotóv Polski oraz ziem historycsnie z polska zwiazanych, t. I : 960-1501, Varsovie, 1974, p. 63.

${ }^{67}$ Philip Grierson, Lucia Travaini, Medieval European Coinage, 14 Italy (III) (South Italy, Sicily, Sardinia), Cambridge, 1999, p. 129, et Catalogue, n² 297-302.

68 Rénier CHALON, Recherches sur les monnaies des comtes de Namur, Bruxelles, 1860, p. 38-39.

${ }^{69}$ D.M. MetCALF, Coinage of the Crusades and the Latin East in the Ashmolean Museum Oxford, $2^{\text {nd }}$ ed., Londres, 1995, p. 157 cite ce type comme "horse and cross/Agnus dei" avec le commentaire suivant : « the die-engraver evidently thought that he was engraving a horse ».

70 Corpus Nummorum Italicorum, vol. VI : Veneto (Dalmazia-Albania), Rome, 1922, p. 39, n 1-4, pl. IV, 1-2.

${ }^{71}$ Leodegar CORAGGIONI, Münzgeschichte der Schweiz, Genève, 1896, pl. XXXII, 21-26 ; H. MEYER, Die Denare und Bracteaten der Schweiz, Zürich, 1858, p. 79, n 1-6, pl. V, n 94-95.

72 Arthur ENGEL, Ernest LEHR, Numismatique de l'Alsace, Paris, 1887, p. 255, n 283, pl. XLIII, $\mathrm{n}^{\circ} 17$; p. $255, \mathrm{n}^{\circ} 286$, pl. XLIII, $\mathrm{n}^{\circ} 23$ var.

73 Ladislaus Rethy, Corpus nummorum Hungariae, trad. par G. Probst en allemand, Graz, 1958, pl. XIII, $\mathrm{n}^{\circ} 229-230$.

${ }^{74}$ Edmund KOPICKI, Katalog podtsawowych typóv monet i banknotóv Polski oraz ziem historycsnie z polska zwiazanych, t. I : 960-1501, Varsovie, 1974, p 180, n LXXVIII.

${ }^{75}$ P. VAN DER CHIJS, De Munten der voormalige Hertogdommen Braband en Limburg van der vroegste tidjen Tot aan de Pacificatie van Gend, Haarlem, 1851, pl. I, n 13.

${ }^{76}$ D.M. MetCALF, op. cit. n. 71, p. 50. Attribution incertaine au royaume de Jérusalem, Tyr ou Antioche.

77 Carl-Fridriech von POSERN-KLETT, Münzstätten und Münzen der Städte und geistlichen Stifter Sachsens im Mittelalter, Leipzig, 1846, p. 246, n 927, pl. XXVII, ${ }^{\circ} 15$.

78 A. Engel, R. Serrure, Traité de numismatique du Moyen Age, Bologne, Arnaldo Forni, 1980, 
Il faut bien évidemment y ajouter, pour la période suivante, le monnayage d'or français royal des $\mathrm{XIV}^{\mathrm{e}}-\mathrm{XV}^{\mathrm{e}}$ siècles ${ }^{79}$ et les imitations qui en découlèrent.

On constate l'éparpillement de ces monnayages, éparpillement tant géographique que chronologique. Avec un tel corpus il est plus facile de faire des observations que d'en tirer quelques règles de principes. Ainsi, on observe que l'agneau est plus fréquemment tourné à gauche qu'à droite. On peut décliner toute la typologie de chacun des attributs dans toutes ses variantes : pas de nimbe, nimbe simple, nimbe crucigère ; croix haute, étendard, croix vexillifère ; animal regardant devant lui ; tournant la tête vers la croix ; la patte avant repliée sur le pied de la croix ou non ; animal avec une toison ou sans ; etc. Toujours est-il qu'il est difficile de comprendre pourquoi ce type apparaît à tel endroit à une époque donnée, et pourquoi il en disparaît peu après.

L'observation de ce corpus permet de mieux comprendre ce qui pose problème aux numismates lorsqu'ils veulent interpréter le type de Saint-Gilles : celui-ci ne s'inscrit pas assez clairement dans la lignée du corpus ci-dessus décrit, d'un point de vue typologique. Cependant ce corpus n'a de sens qu'a posteriori, pour l'historien, et qu'en réalité cette liste ne constitue pas un corpus, en ce sens qu'aucune de ces monnaies n'est liée à une autre à travers ce processus d'imitation ou d'influence si présent par ailleurs dans la numismatique. Ces émissions apparaissent indépendamment les unes des autres, et il faut aller plus loin dans l'analyse : faire appel à d'autres corpus, faire appel à d'autres supports.

Reprenons d'abord l'argumentation étayant la vision d'une biche sur la moneta egidiensis.

Avant tout, occupons-nous d'une pièce tout à fait étrange qui se trouve illustrée dans les planches du tome II des Monnaies féodales de Poey d'Avant: le n 3715. Le 
quadrupède est représenté couché, sans la croix ${ }^{80}$; or la description qui y correspond dit : «Même type », c'est-à-dire renvoyant à la description de la pièce précédente, qui parle d'un «Agneau pascal, surmonté d'une croix grecque à long pied entre deux points. » Par ailleurs, Poey d'Avant fait référence à une édition antérieure de cette pièce, dans le numéro de 1843 de la Revue numismatique ${ }^{81}$. Quand on se reporte à l'article en question (et à la planche qui s'y rattache), l'animal y est tout à fait comme les autres, debout sur ses pattes, avec une croix. Sur le dessin de 1843, le bas de la monnaie est assez usé, et les pattes sont légèrement estompées. C'est donc au moment de la gravure de la planche des Monnaies féodales qu'une erreur s'est introduite. La pièce telle qu'elle apparaît sur cette planche n'existe nullement. Nous avons alors une série très homogène.

Etudions à présent les arguments opposés à l'Agnus Dei.

1. La forme de l'animal.

On l'a vu, ce n'est pas un argument déterminant, car ce n'est pas ce sur quoi se fondent les artisans médiévaux pour caractériser une figure et la distinguer d'une autre.

Les bractéates de Silésie sont à cet égard extrêmement intéressantes. En effet on y trouve toutes sortes d'animaux ${ }^{82}$, dont un certain nombre de quadrupèdes. Parmi ces quadrupèdes, quelques-uns portent une croix vexillifère, et leur tête est tournée vers l'arrière. Ce ne peuvent être que des Agnus $D e i^{83}$. Mais en dehors de ces deux attributs (croix et tête vers l'arrière), rien ne permettrait de reconnaître l'animal appelé agneau, ou mouton. Le dessin est trop grêle pour cela. Les contraintes techniques de la fabrication des bractéates peuvent contribuer à expliquer un rendu médiocre, l'importance de l'attribut n'en ressort que davantage. En outre, la tête de l'animal n'est

\footnotetext{
${ }^{79}$ A partir de Philippe IV le Bel (Duplessy 1999, $\left.\mathrm{n}^{\circ} 212\right)$.

80 POEY D’AVANT n ${ }^{\circ} 3715, \mathrm{pl}$. LXXXI, n 14.

${ }^{81}$ Cf. $R N 1843$, p. 391, pl. XV, 2.

82 Cf. Edmund KoPICKI, Katalog podtsawowych typóv monet i banknotóv Polski oraz ziem historycsnie z polska zwiazanych, t. I : 960-1501, Varsovie, 1974, p. 167-244.
} 
pas nimbée. Ce sont des quadrupèdes avec cet attribut. Donc ce sont des Agneaux pascals.

2. La tête de l'animal est dirigée vers l'extérieur; aucune de ses pattes avant n'est repliée.

Dans la liste des monnaies à l'Agnus Dei donnée plus haut, seule la bractéate des évêques de Meissen, qui date de la seconde moitié du XIII ${ }^{\mathrm{e}}$ siècle, montre un animal dont la tête n'est pas tournée vers l'extérieur. 12 ateliers sur 13 frappent donc avec un Agneau tourné vers l'intérieur. En cela, le type de Saint-Gilles se distingue clairement du reste. Il est clair que sur les monnaies cet attribut est très présent. Il l'est d'ailleurs sur les autres supports également, quoique de façon moins marquée. Dans l'architecture, on trouve plus facilement des exemples d'Agneau regardant devant soi : sur le tympan de la collégiale de Léon (XII ${ }^{\mathrm{e}}$ siècle), par exemple. Mais même là, les représentations montrent pour plus de neuf dixièmes un animal à la tête tournée vers l'arrière.

La patte avant est un attribut au moins aussi fréquent, sinon davantage, que la tête retournée. Toutefois le cas de l'animal de Saint-Gilles est un peu particulier: si l'on considère le pied de la croix comme l'axe vertical, l'animal est légèrement penché vers l'avant, si bien que sur la plupart des monnaies une de ses pattes avant touche la croix sans pour autant être repliée.

3. La légende circulaire.

La légende qui accompagne la plupart des représentations de l'Agnus Dei dit le plus souvent : Ecce Agnus Dei qui tollit peccata mundi. Cela amène une certaine réticence à voir une telle figure théologique entourée sobrement de : ONOR SCI EGIDII. Comme on l'a vu, cette légende ne désigne pas l'ermite, mais le comté. Elle n'est donc pas à mettre au crédit de la biche. Doit-elle être un argument contre l'Agneau ?

Contrairement aux autres supports, sur la plupart des monnaies de la période qui nous

\footnotetext{
${ }^{83}$ Cf. fig. 5.
} 
intéresse la légende la plus fréquente entourant l'Agneau pascal n'est pas la citation évangélique. D'abord, la plupart d'entre elles ne sont entourées d'aucune légende, aucune inscription : sur les bractéates de Silésie et de Saint-Gall, sur les deniers de Thienen, etc. Souvent aussi elle est illisible, comme à Tripoli, Bressanone ou en Bohême. Bref, sur toutes les monnaies mentionnées plus haut, seules celles de Namur et de Neisse (et celles de Chivasso, plus tardives) ${ }^{84}$ comportent le mot Agnus en légende, et seules les deux premières reprennent la proclamation de Jean-Baptiste.

En outre à Saint-Gilles, le choix des légendes Honor sancti Egidii au droit et Ramundus ou Anfos comes au revers s'expliquent: ce même schéma se retrouve rigoureusement à Toulouse, où le comte monnaie en tant que comte de Toulouse. Guillaume IV, Raymond IV et Bertrand ont fait de même avant Alphonse-Jourdain ${ }^{85}$, ainsi que ceux qui lui ont succédé. Par exemple, sur les deniers de Raymond V de Toulouse, successeur d'Alphonse-Jourdain, on lit au droit ${ }^{86}$ : TOLOSA CIVI ; et au revers (autour de la croix) : RAMON COMES. Le même système est très naturellement employé dans l'atelier de Saint-Gilles : dénomination du lieu au droit, et titulature du comte au revers.

L'Agneau pascal n'est pas entouré de l'inscription qui le désignerait comme tel, parce qu'une monnaie doit être fonctionnelle, et que le comte de Saint-Gilles voulait y mettre certaines informations qui excluent, par manque de place, le message évangélique. Rappelons aussi que, pour les seules monnaies ecclésiastiques (a priori plus portées à placer des inscriptions de nature religieuses), sur 68 types différents frappés au XII ${ }^{\mathrm{e}}$ siècle dans le royaume, seule la moitié comporte des termes à caractère religieux. Et ce sont exclusivement les noms des saints patrons des églises abbatiales ou cathédrales. En France, les phrases entières n'apparaissent que sous saint Louis, avec la création du gros

\footnotetext{
${ }^{84}$ Corpus Nummorum Italicorum, vol. II : Piémont-Sardaigne, Rome, 1911, p. 209, pl. XVIII, 3.

85 POEY D’AVANT n ${ }^{\circ} 3677$ et suiv.

${ }^{86}$ POEY D’AVANT n ${ }^{\circ} 3702$.
} 
d'argent, et ne se répandent vraiment que dans la seconde moitié du XIV siècle.

\section{Pro Agno Dei}

En dehors de la question des attributs propres à l'Agnus Dei, dont il a été question, revenons sur des arguments proprement numismatiques qui incitent à penser qu'il s'agit bel et bien à Saint-Gilles d'un Agneau pascal.

Dans son article consacré à l'Agnus Dei comme type monétaire, Michel Dhénin ${ }^{87}$ note que l'évolution et le développement de ce type se font sans continuité chronologique ou géographique. On ne peut que constater une suite d'apparitions et de disparitions, sans qu'on puisse établir une chaîne d'influence d'une monnaie à l'autre, d'un monnayage à l'autre. L'Agneau est frappé dans un atelier sans qu'on sache pourquoi, et en disparaît sans plus de raison, et sans avoir été imité par un atelier voisin (si l'on excepte le cas des monnaies royales françaises de la fin du Moyen Age).

Puisque ce type apparaît néanmoins, c'est donc nécessairement qu'il est véhiculé par un support autre que monétaire. Mais s'il est régulièrement adopté par les monnayeurs, ce véhicule est alors probablement assez proche de l'objet monétaire lui-même, pour susciter ainsi des inspirations aussi indépendantes les unes des autres.

Ce support est sans doute double: d'une part des Agnus Dei proprement dits, ces objets de cire dispensés à Rome au moment de la fête de Pâques; d'autre part les nombreuses reproductions de cet objet par des orfèvres, qui en font de petits objets précieux, de la taille d'un sceau. Les sceaux à l'Agnus Dei eux-mêmes, en très grand nombre, peuvent inspirer les graveurs. Comme on l'a vu, de nombreux sceaux de la région comportent un Agnus Dei. Bien sûr, ils apparaissent généralement plus tard. Néanmoins il semble que la région en produit plus que d'autres. En Angleterre au début du XI ${ }^{\mathrm{e}}$ siècle, Aethelred II frappe aussi au même type. Certains chercheurs ont associé

\footnotetext{
${ }^{87}$ Michel DHENIN, op. cit. n. 60, p. 163-177.
} 
l'apparition de ce type à « une nouvelle vogue au début du $\mathrm{XI}^{\mathrm{e}}$ siècle pour l'Agnus Dei. ${ }^{88}$ Il y a peut-être de cela lors de la création du monnayage de Saint-Gilles un peu plus tard.

Replaçons à présent la monnaie de Saint-Gilles dans le corpus des monnaies frappées dans le royaume entre le $\mathrm{X}^{\mathrm{e}}$ et le $\mathrm{XV}^{\mathrm{e}}$ siècle. Supposer une biche de saint Gilles au sein de ce corpus signifie voir un apax, une situation sans équivalent.

On trouve en effet dans les royaumes de l'Europe de l'Est, en terre d'Empire certains seigneurs ou rois qui frappent des pièces reproduisant tout un bestiaire, avec des variétés d'oiseaux, des bovins, des animaux sauvages ou imaginaires, et toutes sortes de scènes. La frappe tchèque ${ }^{89}$ est à cet égard révélatrice. La typologie y est extrêmement mouvante. La France à l'inverse a conservé plus longtemps l'héritage carolingien, et en a gardé définitivement son influence. Si les types du IX ${ }^{\mathrm{e}}$ siècle disparaissent, un principe qui « réglemente» le choix de ces types persiste: on ne trouve sur les monnaies du royaume que des représentations statiques. Si l'on excepte quelques pièces royales tardives $\left(\mathrm{XV}^{\mathrm{e}}\right.$ siècle) montrant saint Michel terrassant le dragon, et la Visitation, aucune image monétaire n'a de mouvement : ce sont des symboles, des emblèmes, des signes figés. Il ne s’y passe rien : bustes, objets chargés d'une connotation forte, inscriptions, etc. Les seuls animaux sont héraldiques: le lion et le léopard, l'aigle (Valence), l'hermine (Bretagne), le cheval (type sigillographique sur le franc à cheval, en 1360), l'Agneau pascal (représenté adulte, sur les moutons d'or royaux), la vache du Béarn ${ }^{90}$, le dauphin du Viennois.

De cette liste d'animaux, l'image comportant le plus de mouvement est celle du franc à cheval, qui est en réalité une figure complètement figée, réglementée, jusque dans la

${ }^{88}$ Dolley, cité par E. OKASHA, J. O’Reilly, «An Anglo-Saxon Portable Altar : Inscriptions and Iconography », Journal of the Warburg and Courtauld Institute, 1984, n 47, p. 32-51.

${ }^{89}$ F. CACH, Nejstarši Cešké Mince, Prague, Ceská Numismatická Spolecnost, 1972, 3 tomes. 
pénétration codifiée des sabots de l'animal dans la légende pour renforcer l'impression du mouvement.

A l'inverse, la représentation d'une biche, fût-elle celle de saint Gilles, n'est absolument pas codifiée. Cette image serait tirée d'une scène de la Vita sancti Aegidii, à un instant précis de l'existence du saint. Si cela se produit en terre d'Empire ${ }^{91}$, c'est un procédé qui n'a pas d'équivalent dans le royaume de France du XII ${ }^{\mathrm{e}}$ siècle. En outre, toutes les représentations de saints sont sous forme de bustes ${ }^{92}$, de reliquaires ${ }^{93}$ (éventuellement de bustes-reliquaires) ou d'objets de dévotion ${ }^{94}$. On ne trouve ni allusion à un épisode de la vie du saint, ni aucun animal. Si le graveur avait mentionné saint Gilles par une image, il aurait suivi sur ce point les mêmes règles qu'ailleurs.

En outre, il lui aurait fallu choisir un attribut à cette biche, pour qu'il soit clairement établi qu'il s'agissait de celle de saint Gilles. Comme on l'a vu, une croix haute ne peut remplir ce rôle.

Revenons pour finir sur la forme de l'animal : Et si pourtant l'animal représenté était tout de même ressemblant? L'animal ne ressemble-t-il pas malgré tout à un agneau ? Reprenons-le en détail. L'animal ne ressemblerait pas à un agneau pour trois raisons : il n'a pas de toison, comme sur les pièces d'or royales du XIV ${ }^{\mathrm{e}}$ siècle ; ses pattes et son cou sont trop longs; il a une longue queue.

Le dernier point, la longue queue, est erroné. Car à l'époque les moutons, précisément, ont une longue queue. Jean-René Gaborit la qualifie de «queue grasse » quand il décrit un linteau conservé au Musée du Louvre ${ }^{95}$ et montré à l'occasion de

\footnotetext{
90 PoEY D’AVANT n ${ }^{\circ} 3249$.

${ }^{91}$ Lapidation de saint Etienne, partage de son manteau par saint Martin.

92 Mende, Vienne, Saint-Martial de Limoges, etc.

93 Besançon.

94 Saint-Médard de Soissons.

95 Paris, Musée du Louvre, Département des sculptures (nº RF. 1673). Cf. fig. 7.
} 
l'exposition La France romane ${ }^{96}$. Notant la présence de cette «queue grasse », il en conclut même « une certaine précision zoologique ». A l'inverse, justement, les biches n'avaient pas davantage de «queue grasse » (ou longue) au XII ${ }^{\mathrm{e}}$ siècle qu'au XX ${ }^{\mathrm{e}}$, ainsi qu'on le constate sur les représentations de scènes de la vie de saint Gilles ${ }^{97}$.

L'absence de toison est normale pour un agneau. Cela le différencie du mouton. Ce sont bien plutôt les graveurs du roi de France qui se sont fourvoyés ${ }^{98}$.

Quelques éléments demeurent donc qui maintiennent une certaine suspicion sur la véracité de cet agneau : la longueur des pattes et du cou, pour ce qui est de la forme de l'animal. Et ces attributs que sont la tête retournée et la patte avant repliée. Toutefois l'absence de ces attributs se rencontre parfois. Elle ramène quoi qu'il en soit à la question du modèle qui a servi pour ces monnaies. Car finalement, il se pourrait bien que la représentation de l'agneau soit relativement ressemblante. Mais il n'en est pas moins certain que le graveur n'a pas utilisé un agneau vivant (ou mort) pour faire son travail. Il s'est sans nul doute inspiré de l'œuvre d'un autre artisan. Or on trouve dans un manuscrit une enluminure dont l'animal ressemble beaucoup à celui que l'on voit à Saint-Gilles. Il s'agit du Commentaire de l'Apocalypse de Beatus conservé à la Pierpont Morgan Library, où le dessin d'un Agnus $D e i^{99}$. Sans prétendre à une imitation directe de l'enluminure par le graveur de coin, on pourrait supposer du moins une influence commune. Un simple coup d'œil sur cette enluminure permet de voir la ressemblance frappante entre l'Agneau de l'Apocalypse qui y est dessiné, et l'animal des monnaies de Saint-Gilles. L'enluminure date du milieu du $\mathrm{X}^{\mathrm{e}}$ siècle, et a été composée dans le nord de l'Espagne. On est assez proche de Saint-Gilles, et pas bien loin de la Première

\footnotetext{
96 La France romane : au temps des premiers Capétiens (987-1152). Catalogue de l'exposition La France romane, Paris, Musée du Louvre, 10 mars-6 juin 2005, Paris, Hazan-Musée du Louvre, p. 111

97 Peintures sur les murs de l'église Saint-Nicolas de Civray, ou à Largny-sur-Automne, dont les reproductions numérisées se trouvent dans la base Mérimée du Ministère de la Culture.

98 En réalité, ils n'ont fait qu'accentuer les attributs de l'Agneau. Ce phénomène visant à une clarté plus grande de la figure représentée est déjà notable aux IV-VI siècles, selon le Dictionnaire de théologie catholique, déjà cité.
} 
Croisade...

Si l'image des monnaies de Saint-Gilles n'a eu ce modèle, elle a dû en avoir un autre. Il paraît indispensable de supposer l'existence d'un modèle antérieur, car sur la monnaie de Saint-Gilles se trouve sans cela un élément inexplicable : les deux globules de part et d'autre de la croix. Ils n'ont pas de sens sur la monnaie. Mais on peut penser qu'ils en avaient un sur l'image d'origine, et qu'ils ont été reproduits et simplifiés. Sur l'enluminure du Commentaire de l'Apocalypse, l'Agneau tient devant lui un livre. Que serait devenu ce «livre» sur une copie de l'enluminure ? Le graveur de Saint-Gilles aurait pu par exemple utiliser une image où la croix aurait été accostée du soleil et de la lune, et les aurait restitués aussi bien que possible. Quelle que soit leur origine, ces deux globules suggèrent fortement l'existence d'une image initiale. Si l'artisan n'y a pas été un grand observateur de la nature, s'il n'a pas voulu rendre avec précision la morphologie d'un agneau, il est difficile d'en faire ensuite le reproche au graveur de l'atelier monétaire.

Plusieurs points doivent donc être impérativement considérés comme définitifs. D'abord, la forme d'un personnage (humain ou animal) ne saurait être plus importante que ses attributs pour l'identifier. Etant donné les pratiques dans les ateliers monétaires pour le choix des types, la représentation d'une biche tirée de la vie de saint Gilles est unique, et doit sûrement étonner: d'abord parce qu'une scène de Vita serait un cas unique dans le royaume ${ }^{100}$, ensuite parce que, avec la présence d'une croix haute en travers du corps de l'animal, la confusion créée avec l'Agnus Dei est trop forte.

Parallèlement, l'Agnus Dei est une représentation très fréquente, l'iconographie est

\footnotetext{
${ }^{99}$ Cf. fig. 8 .

${ }^{100}$ Une des principales exceptions à cette règle serait peut-être la lapidation de saint Etienne sur un demi-gros de l'évêque de Metz Adhémar de Monteil (1327-1334). Cf. A. ENGEL, R. SERrure, Traité de numismatique du Moyen Age, Paris, 1891-1905, fig. 1623. Mais cette scène est extrêmement courante, et sa représentation figée. En outre, Metz est en terre d'Empire.
} 
relativement stable depuis plusieurs siècles lorsque apparaît le monnayage de SaintGilles, avec un certain nombre de variantes dans les détails cependant. Toutefois un de ses attributs est systématique : l'Agneau se trouve dans un cercle.

Ses autres attributs sont absents sur la monnaie de Saint-Gilles : pas de nimbe, pas de toison, pas de patte repliée, pas de tête retournée. Si bien que, Agneau ou biche, le type de Saint-Gilles est difficile à interpréter.

Le fait que l'Agneau soit dans un cercle souffre de nombreuses exceptions, en particulier lorsqu'un Agneau apparaît sur un sceau : il s'agit alors souvent d'un sceau ecclésiastique, et les sceaux épiscopaux sont souvent en fuseau. En conséquence, on trouve de nombreux sceaux en fuseau avec un Agnus $D e i^{101}$. Mais on trouve également de nombreux cas où l'Agnus Dei est renvoyé sur le contre-sceau, qui est rond même lorsque le sceau est ovale.

Cette caractéristique, qui fait de l'Agnus Dei une image proprement circulaire, semble secondaire aux iconographes. Elle est fondamentale. En effet, elle est liée à l'histoire de la propagation de cette figure, sur des supports très spécifiques : les Agnus Dei de cire. Progressivement, cette cause a été, semble-t-il, oubliée. La preuve en est que l'on rencontre aujourd'hui, dans les églises, des représentations de l'Agneau pascal tenant une croix vexillifère, où le cercle autour de l'animal est brisé en deux verticalement, comme une hostie. Faute de comprendre le sens originel, les artistes lui en trouvent un nouveau : et c'est ainsi que l'iconographie évolue.

Toujours est-il qu'au XII ${ }^{\mathrm{e}}$ siècle, l'Agneau pascal et le cercle étaient pour ainsi dire indissociables, ou peu s'en faut.

La présence de l'Agneau sur les « disques » est tellement forte qu'il devient curieux que l'Agneau pascal ne se retrouve pas de façon beaucoup plus systématique sur les monnaies médiévales. Il est partout (sur les sceaux, les reliures orfévrées), sauf là. Sa

${ }^{101}$ Cf. les deux sceaux hospitaliers déjà mentionnés, appendus à une indulgence de 1410 (E. J. KING, 
présence à Saint-Gilles est somme toute beaucoup moins étonnante que son absence partout ailleurs.

Il est difficile de déterminer si c'est là une question légitime : s'il est déjà risqué pour un historien de prétendre expliquer l'emploi d'une image en un endroit, peut-il oser chercher les raisons de son absence ailleurs?

op. cit $\mathrm{n} .53 .$, pl. XX, 5-6). 


\section{ANNEXE}

\section{Description des planches}

\section{Fig. 1 : Denier de Saint-Gilles}

ONOR SCI EGIDI Quadrupède surmontée d'une croix haute entre deux globules. R. : + ANFOS CONES Croix pattée dans un grènetis.

Billon. 1,01 g. $12 \mathrm{~h}$.

Kunsthistorisches Museum de Vienne, Münzkabinett, Fonds français, $\mathrm{n}^{\circ} 22.095 \mathrm{a} \alpha$.

\section{Fig. 2 : Denier à l'Agneau de Bohême}

Monnaie de Svatopluk (1095-1107).

+ SVA[TOPLV..]SO Tête barbue de face, dans un grènetis, surmonté d'une coiffe pointue.

R. : WENCEZLVA Agnus Dei à droite, tête tournée vers l'intérieur, nimbé, tenant une croix haute avec une patte avant repliée.

František CACH, Nejstarš̀ Cešké Mince, Prague, Ceská Numismatická Spolecnost, 1972, t. II, nº 439.

Fig. 3 : Denier du roi de Hongrie Béla IV (1235-1270) :

+ REX BELA QVARTVS Agneau pascal à gauche, la tête nimbée tournée à droite, tenant une croix haute, dans un grènetis.

R. : VNGA - RIE Roi en majesté, assis, couronné, tenant un sceptre et un globe, coupant la légende.

L. RÉTHY, Corpus nummorum Hungariae, trad. par G. Probst en allemand, Graz, 1958, pl. XIII, $\mathrm{n}^{\circ} 229$.

Fig. 4 : Obole du roi de Hongrie Béla IV (1235-1270) :

REX VNGARIE Agneau pascal à gauche, la tête nimbée tournée à droite, tenant une croix haute, dans le champ.

R. : Buste du roi de face, couronné, tenant un sceptre et un globe.

L. RÉTHY, Corpus nummorum Hungariae, trad. par G. Probst en allemand, Graz, 1958, pl. XIII, $n^{\circ} 230$.

La figure du roi est presque omniprésente sur les monnaies de Béla IV. Ce n’est pas le cas de l'Agnus Dei, qui n'apparaît ici que sur une émission. On trouve sur ses monnaies de nombreux autres animaux, réels ou merveilleux. 
Le roi Andreas II (1290-1301) garde le type de l'Agnus Dei sur au moins cinq pièces différentes. Aucune légende circulaire n'entoure le type. L’Agneau est toujours nimbé, tient une croix haute vexillifère, et sa tête est tournée vers l'intérieur (en revanche le corps peut être tourné à droite ou à gauche) ${ }^{102}$.

Fig. 5 : Bractéate de Silésie (XIII ${ }^{\mathrm{e}}$ siècle) :

Agneau pascal tenant une croix vexillifère.

Edmund KOPICKI, Katalog podtsawowych typóv monet i banknotóv Polski oraz ziem historycsnie z polska zwiazanych, t. I : 960-1501, Varsovie, 1974, p. 180, n² LXXVIII.

Fig. 7 : Monnaie de Thienen, ou Tirlemont $\left(\mathrm{XI}^{\mathrm{e}}-\mathrm{XII}{ }^{\mathrm{e}}\right.$ siècle $)$ :

Agneau pascal à droite, tête tournée vers l'intérieur, tenant une croix haute avec une patte avant repliée.

R. : Croix évidée ; trois traits en 1 et 4 , un globule dans un cercle et un globule en 2 et 3.

P. VAN DER CHIJS, De Munten der voormalige Hertogdommen Braband en Limburg van der vroegste tidjen Tot aan de Pacificatie van Gend, Haarlem, 1851, pl. I, nº 13.

Fig. 7 : Agneau pascal dans un linteau, accompagné de l'inscription : + ECCE DEI MISERENS AGN[VS] / MOENIA SISTENTES FRA[TRES].

Languedoc-Roussillon (v. 1100). Marbre blanc.

Paris, Musée du Louvre, département des sculptures ( $n^{\circ}$ RF. 1673)

La France romane. Au temps des premiers Capétiens. Catalogue de l'exposition du Musée du Louvre, 10 mars-6 juin 2005, Paris, Hazan-Musée du Louvre, p. 111, nº 65.

Fig. 8: Agneau pascal tenant entouré des quatre animaux de l'Apocalypse, représentant chacun des Evangélistes. Commentaire de l'Apocalypse de Beatus de Liébana. Pierpont Morgan Library, ms 644,

102 Corpus nummorum Hungariae, pl. XVIII, n $364-368$. 
fol. $87^{103}$

Création : Nord de l'Espagne, près de Tábara (royaume de Léon), v. 940-945.

Style mozarabe.

L'Agneau tient un livre devant lui.

103 La description de cette enluminure, sans illustration, est disponible sur la base de données de la bibliothèque, intitulée : CORSAIR. Je tiens à remercier vivement Inès Villela-Petit pour son aide dans l'identification de cette enluminure. 
PLANCHES

-
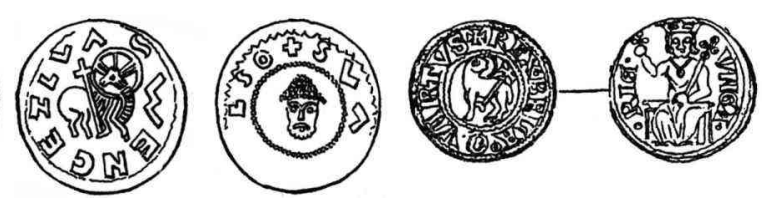

1

2

3

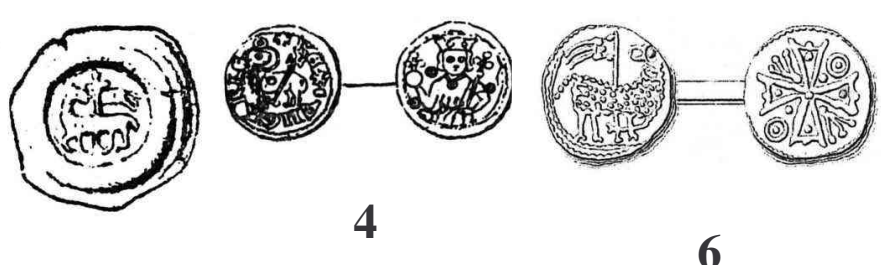

5

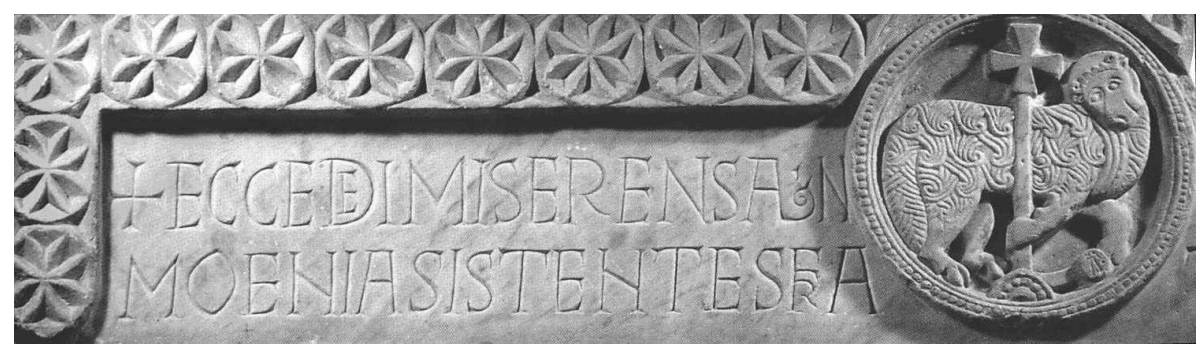




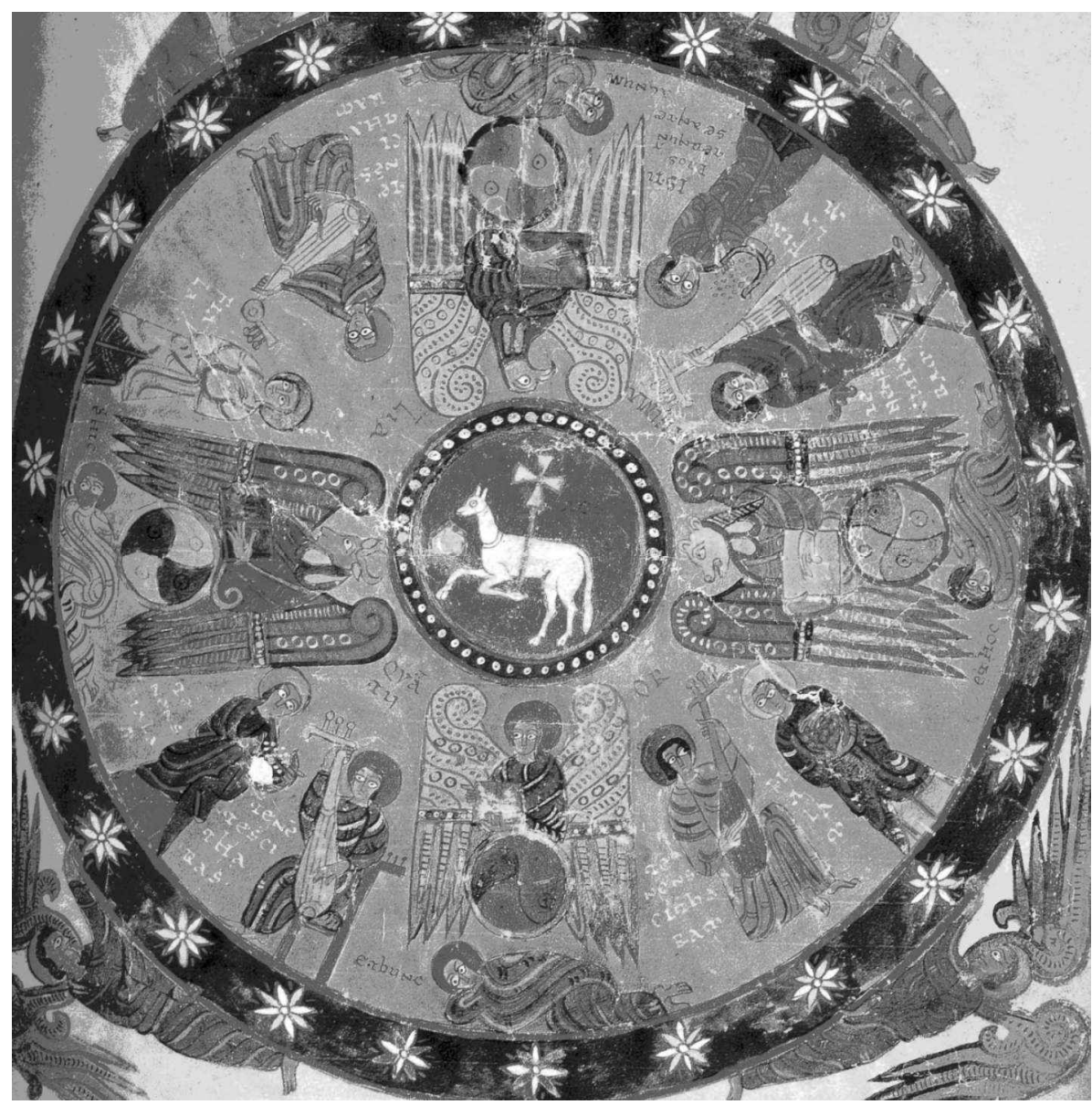

